\title{
Pratiques
}

Linguistique, littérature, didactique

169-170| 2016

Enseignement/apprentissage de la langue, des textes et des discours. 40\&nbspans de Pratiques

\section{Que faire de la grammaire et comment en faire?}

What to do with Grammar and how to do it?

Jean-Paul Bronckart

\section{(2) OpenEdition}

Journals

Édition électronique

URL : http://journals.openedition.org/pratiques/2959

DOI : 10.4000/pratiques.2959

ISSN : 2425-2042

Éditeur

Centre de recherche sur les médiations (CREM)

Référence électronique

Jean-Paul Bronckart, "Que faire de la grammaire et comment en faire? », Pratiques [En ligne],

169-170 | 2016, mis en ligne le 30 juin 2016, consulté le 14 novembre 2019. URL : http://

journals.openedition.org/pratiques/2959; DOI : 10.4000/pratiques.2959

Ce document a été généré automatiquement le 14 novembre 2019.

(c) Tous droits réservés 


\title{
Que faire de la grammaire et comment en faire?
}

What to do with Grammar and how to do it?

\author{
Jean-Paul Bronckart
}

1 Chez les enseignants de langue, chez leurs formateurs et dans le milieu de la didactique du français en général, la grammaire et son enseignement ont été et demeurent les objets d'un questionnement comportant généralement trois strates. Les approches grammaticales s'adressent-elles à un véritable domaine de réalité langagière, et si oui quels sont la nature et l'empan de ce domaine? La maitrise de notions et de règles grammaticales peut-elle être utile au développement des capacités langagières des élèves, et si oui, quel modèle de référence adopter pour conceptualiser ces notions et ces règles? Quel que soit le cadre de référence choisi, quelle place donner à l'enseignement grammatical dans les programmes scolaires, et quelle méthodologie mettre en œuvre pour en assurer l'efficacité ?

2 Pour nombre d'enseignants et de formateurs, ce questionnement aurait émergé dans les années 70 pour des raisons à la fois théoriques et didactiques, à savoir d'un côté sous l'effet du déclin relatif des approches structuralistes face à la floraison des approches énonciatives, pragmatiques et discursives/textuelles, et d'un autre côté en raison d'un réaménagement des objectifs assignés à l'enseignement du français, accordant une nette priorité au développement des capacités d'expression orale et écrite. Ce diagnostic n'est que partiellement correct, dans la mesure où, comme nous le montrerons dans les deux premières parties de cette contribution, d'une part, le problème grammatical a été posé, et de manière particulièrement rude, dès l'instauration de l'École publique obligatoire dans le dernier quart du XIX ${ }^{e}$ siècle, et, d'autre part, les projets de rénovation élaborés et mis en œuvre dans les années 70-80 comportaient des propositions de refondation de l'enseignement grammatical plus substantielles et précises que celles ayant trait au domaine de l'expression orale et écrite.

3 Il n'en demeure pas moins que les trois ordres de questions ci-dessus évoqués demeurent d'actualité, et dans la $3^{\mathrm{e}}$ partie nous donnerons quelques exemples de 
l'indécision et de la confusion qui se sont instaurées (et qui s'accroissent) aujourd'hui sur le plan des objectifs, du corpus notionnel et de la méthodologie de l'enseignement grammatical, et nous analyserons les raisons et facteurs ayant généré ce qu'il convient de qualifier de marasme didactique. Nous prendrons alors appui sur une approche théorique inspirée de F. de Saussure pour approfondir le statut de la démarche grammaticale et la nature de l'objet qu'elle vise (partie 4), ce qui nous permettra pour clore (partie 5) de formuler quelques propositions didactiques ayant trait à la clarification des objets, visées et méthodes de cet enseignement et à la place qu'il peut/ doit prendre dans les programmes d'enseignement de la langue première.

\section{Haro sur la grammaire !}

4 S'il est l'auteur d'importants travaux de sémantique et de linguistique comparée, M. Bréal est surtout célèbre chez les didacticiens du français pour son ouvrage de 1872 stigmatisant l'état de l'enseignement en France et proposant un ensemble de principes et de projets d'amélioration qui allaient être en partie concrétisés dans les lois Ferry de 1881-1882 instaurant l'École publique, laïque, gratuite et obligatoire. Dans ce plaidoyer fondateur, M. Bréal, après avoir souligné l'importance de la syntaxe et de sa maitrise par les apprenants, formulait à propos de l'état de l'enseignement ayant trait à ce domaine le sévère commentaire qui suit :

Il semble d'après cela qu'il ne puisse y avoir d'enseignement plus attrayant et plus substantiel que la leçon de grammaire française. D'où vient cependant qu'elle est la plus vide et la plus rebutante de toutes? Si nous consultons nos souvenirs, nous nous rappellerons que la grammaire française était pour nous une source d'ennuis et de dégoûts, et si nos maîtres veulent être sincères, ils conviendront que c'est cette leçon qui, dès les premiers pas, détourne pour toujours de l'école ou décourage de tout effort sérieux une quantité de nos enfants. (M. Bréal, 1872, p. 31-32)

Dans sa longue argumentation ayant trait aux conditions d'un enseignement efficace du français de l'école primaire à l'université, M. Bréal ne proposait nullement de supprimer l'enseignement grammatical, mais bien plutôt de le réformer et de l'articuler efficacement à des démarches d'amélioration des capacités d'expression orale (puis écrite) des élèves. La plupart de ses commentateurs ne retinrent cependant que sa dénonciation de l'enseignement grammatical, ce qui déboucha, dans les dernières décennies du $\mathrm{XIX}^{\mathrm{e}}$, sur un mouvement de contestation radicale dans lequel s'illustrèrent en particulier journalistes et romanciers. À titre d'exemple, R. Frary, essayiste auteur d'un Manuel du démagogue (1884) célèbre en son temps, écrivait ce qui suit dans un ouvrage plaidant par ailleurs pour une suppression définitive de l'enseignement du latin :

Nous savons le français sans nous donner la peine de l'apprendre. Il suffit d'écouter ceux qui parlent bien et de lire des auteurs qui ont bien écrit. Il semble que ce bienfait de la nature nous ait déplu parce qu'il était gratuit. [...] Je voudrais qu'on épargne aux élèves la torture de la grammaire, l'orthographe d'usage leur viendra d'elle-même. Quant aux règles, on les leur enseignera en peu d'heures, lorsqu'ils seront en état de les comprendre. (Frary, 1885, p. 218-219)

6 Et quelques années plus tard, dans Pierre Nozière, A. France tenait un discours aussi virulent :

Je tiens pour un malheur public qu'il y ait des grammaires françaises. Apprendre dans un livre aux écoliers leur langue natale est quelque chose de monstrueux, 
quand on y pense. Étudier comme une langue morte la langue vivante: quel contresens! Notre langue, c'est notre mère et notre nourrice, il faut boire à même. Les grammaires sont des biberons. Et Virgile a dit que les enfants nourris au biberon ne sont dignes ni de la table des dieux ni du lit des déesses. (France, 1899, p. 146-147)

$7 \mathrm{Au}$ cours du $\mathrm{XX}^{\mathrm{e}}$ siècle, les discours critiques se sont poursuivis avec une belle régularité, la plupart émanant cependant désormais de linguistes et/ou de pédagogues qui défendaient leur position de manière plus sobre et plus argumentée. Ainsi F. Brunot, lui-même grammairien, contestait principalement la manière dont les ouvrages de grammaire étaient conçus et rédigés, et critiquait vivement le type de pédagogie de la langue qu'ils engendraient :

Ce que beaucoup se demandent, c'est si l'enseignement de la grammaire, tel qu'il est, mène au but que l'on se propose, et je crois qu'ils ont tout à fait raison de s'interroger et de douter, car [...] ce n'est pas qu'on enseigne trop peu la grammaire, c'est qu'on l'enseigne mal: abstractions incompréhensibles, définitions prétentieuses et néanmoins plus souvent vides, règles fausses, énumérations indigestes, il n'y a qu'à feuilleter quelques pages d'un manuel pour trouver des spécimens variés de ces fautes contre la raison, la vérité et la pédagogie. (Brunot, 1908, p. 3)

8 Et plus tard, sous le célèbre titre Et si la grammaire était inutile?, C. Freinet a soutenu que la grammaire enseignée était un résidu de la scolastique et de l'enseignement du latin, et a stigmatisé son statut d'ensemble de règles d'un écrit normé et dépassé, ainsi que la méthodologie déductive (apprendre des règles puis les appliquer) en usage dans son enseignement :

L'étude de la grammaire était peut-être une nécessité dans une période aujourd'hui dépassée. A mode nouveau de vie, techniques nouvelles d'acquisition et de milieu. La question peut se poser aujourd'hui : «Et si la grammaire était inutile ?» [...] On a cru longtemps [...] qu'il n'y a pas d'autre moyen d'apprendre correctement la langue française que d'en apprendre au préalable les règles. Il y a là une grande erreur, dont il faut voir la généralisation et la perpétuation dans les pratiques scolastiques, détachées de la vie et sans résonance sur cette vie. (Freinet, 1951)

Enfin, à partir des années 60-70, la plupart des linguistes et formateurs d'enseignants ont reformulé les critiques portant sur le sociolecte visé par les grammaires, ainsi que sur la finalisation orthographique et la méthodologie de leur enseignement, mais y ont adjoint des arguments plus fondamentaux ayant trait à la conception de l'essence même du langage. À savoir, comme l'ont relevé notamment E. Roulet (1972) et H. Huot (1981), la perpétuation d'une approche représentationnaliste conduisant à définir les classes grammaticales par les sortes de choses qu'elles représentent et à désigner les structures syntaxiques par les opérations cognitives qu'elles sont censées traduire, approche articulée à une conception fixiste et universalisante du langage sousestimant, voire niant, la diversité et la dynamique des langues naturelles.

\section{2. À la recherche d'une modernité grammaticale}

Pour un ensemble de raisons sociopolitiques et économiques que nous ne pourrons commenter ici, c'est dans le courant des deux décennies ci-dessus évoquées que les instances faitières de l'éducation ont finalement accepté de prendre en considération les critiques quasi séculaires adressées à la grammaire et ont adopté le principe d'une 
réforme globale des modalités d'enseignement de la langue française dont le Plan Rouchette ${ }^{1}$ a constitué le socle fondateur.

De manière générale, ce plan proposait une démarche pédagogique centrée sur des activités créatives visant l'enrichissement des capacités d'expression orale et écrite (pôle «libération»), articulées à des activités de réflexion guidée visant à la construction de connaissances relatives aux régularités d'organisation de la langue (pôle «structuration »). S'agissant de ce second pôle, la méthodologie préconisée se déployait en deux phases: introduire d'abord une démarche de grammaire implicite, constituée d'exercices structuraux (empruntés à la didactique du français langue étrangère) dans lesquels les élèves auraient à réaliser, sans conceptualisation, des transformations majorantes ou correctives de leurs énoncés; ensuite une démarche explicite consistant en activités de manipulation d'énoncés (minutieusement décrites par H. Romian, 1973) devant permettre aux élèves de découvrir et de conceptualiser les notions et règles de base de la syntaxe du français. La planification des contenus se caractérisait quant à elle par un abandon de la logique traditionnelle préconisant un parcours du plus simple au plus complexe et par l'adoption d'une progression inverse abordant successivement: la phrase, en tant qu' «unité de discours", avec les transformations qu'elle est susceptible de subir (négation, interrogation, exclamation); les groupes ou syntagmes attestables dans toute phrase, avec les unités linguistiques qui s'y trouvent organisées; les unités singulières, dont certaines sont susceptibles de se substituer aux groupes; les fonctions grammaticales que peuvent assurer les groupes ou les unités.

12 S'il a inspiré la plupart des réformes entreprises ensuite dans l'ensemble de la francophonie, le Plan Rouchette a trouvé un écho particulier en Suisse romande, dont les autorités ont promu un ensemble de travaux visant à la modernisation de l'enseignement de la langue maternelle: l'élaboration d'une méthodologie générale intégrant et coordonnant les traditionnelles composantes d'expression, grammaire, vocabulaire et conjugaison (cf. Besson et al., 1979) ; la production de grammaires et d'autres moyens d'enseignement adaptés aux différents niveaux de la scolarité (cf. Bronckart \& Besson, 1988 ; Besson et al., 1990 ; Genevay, 1994) ; la mise en place d'un dispositif de suivi et d'évaluation des effets de l'implémentation des innovations, assurés par des formateurs, linguistes et pédagogues réunis dans le Groupe Bally².

Ces tentatives de mise en œuvre d'une forme de modernité grammaticale se sont inscrites de fait dans une épistémologie de type empiriste, posant que les notions et règles de grammaire doivent être conceptualisées sur la base de l'analyse des caractéristiques propres de la langue française, et non plus en tant que reflets présumés d'évènements, de relations ou d'états relevant d'une « logique du monde » à caractère universel. Et cette prise de distance à l'égard de la conception représentationnaliste s'est naturellement accompagnée de l'acceptation du caractère dynamique (changeant) des langues naturelles, ainsi que de la prise de conscience de l'extrême diversité des propriétés de ces dernières et de la variété des sociolectes qui les composent.

14 Ces tentatives se sont caractérisées aussi, sur le plan de l'identification et de la dénomination des entités linguistiques, par l'instauration d'une démarche d'abord fondée sur des procédés (manipulations) et critères syntaxiques; ce qui n'excluait pas d'examiner par ailleurs les valeurs ou fonction sémantiques que pouvaient prendre ces entités (valeur de temps ou de lieu ; fonction d'actant ou de patient), mais à la condition 
de clairement dissocier ces deux modes d'analyse. Elles se sont caractérisées encore, sur le plan méthodologique, d'un côté par la volonté de faire porter les exercices proprement grammaticaux sur des segments de textes authentiques et de les articuler ce faisant aux activités relatives à l'expression, d'un autre côté par la mise en place de procédés de guidage visant à développer les raisonnements inductifs des élèves (cf. Bulea Bronckart, 2015 ; Masseron, 2001).

\section{Le marasme contemporain et ses origines}

Trois et bientôt quatre décennies après la mise en œuvre des projets d'innovation qui viennent d'être évoqués, le bilan n'est guère réjouissant, et si, en raison de l'extrême diversité des pratiques d'enseignement grammatical attestables dans les différentes communautés francophones (ainsi qu'au sein de ces dernières), un bilan rigoureusement documenté ne peut être établi, quelques constats généraux peuvent néanmoins être formulés.

L'élément le plus apparent est le spectaculaire désordre notionnel qui s'est progressivement instauré et qui continue de se développer sous l'effet de divers facteurs (cf. De Pietro, 2010). Tout d'abord, alors que certaines communautés (dont la Suisse romande) s'en sont tenues à des appellations découlant d'analyses strictement syntaxiques (par exemple, pour les fonctions grammaticales, introduction des notions de complément de verbe, de complément de phrase et de modificateur), d'autres (la France en particulier) ont maintenu des appellations issues d'analyses mélangeant critères syntaxiques et "sémantiques" (pour les mêmes fonctions, notions de complément d'objet et de compléments circonstanciels de temps, de lieu, de manière, etc.). Ensuite, au sein même des courants prônant un approche résolument moderniste, les décisions d'adaptation/transposition n'ont guère été coordonnées, ce qui a donné lieu à des situations de quasi-concurrence de termes censés désigner la même réalité, comme en atteste par exemple la coexistence, pour désigner la structure canonique des phrases, des expressions " phrase P ", " phrase de base », « modèle P », etc. (cf. Elalouf, 2014). Enfin, les réorientations successives de politique éducative qui se sont manifestées dans l'ensemble des communautés ont produit parfois des décisions proprement insensées, dont la plus remarquable est sans doute la disparition de la notion de "groupe prépositionnel » décrétée en Suisse romande sur le conseil de certains « experts » et dont les effets secondaires sont quasi ubuesques. L'exemple qui précède est en réalité une manifestation extrême (heureusement rare) d'un deuxième facteur de désordre, constitué par les changements d'orientation dont témoignent régulièrement les documents officiels, changements qui s'opèrent la plupart du temps sans véritable argumentation ou justification, et sur des parties seulement des plans d'études ou des autres documents de référence, avec le surcroit d'hétérogénéité qui en découle inévitablement.

Sur le plan méthodologique, on observe la persistance, dans la plupart des manuels en usage, d'activités d'identification/dénomination de fonctions grammaticales, ou de complétion de structures (par " exercices à trous ", cf. Chiss \& Filliolet, 1982) qui sont quasi identiques à celles que proposait $\mathrm{M}$. Grevisse dans ses Exercices sur la grammaire française (1941). Par ailleurs, si l'on observe de-ci de-là des tentatives d'intégration des activités grammaticales au sein de leçons portant sur l'organisation des textes, les conditions et limites de ce type d'intégration ne sont que rarement suffisamment 
précisées (cf. Bulea Bronckart, 2014), ce qui nuit à leur efficacité et à leur pérennité. Par ailleurs enfin, on n'observe qu'exceptionnellement les démarches de guidage qui permettraient de faire émerger chez les élèves des raisonnements grammaticaux véritablement inductifs.

Les raisons de ce marasme grammatical tiennent bien sûr à la volatilité des décisions de politique éducative évoquée plus haut. Mais le marasme découle aussi d'un autre facteur d'ordre politique et économique, celui du champ libre largement laissé aux éditeurs de moyens d'enseignement du français. Lorsque la rénovation a été engagée, diverses maisons d'édition ont entrepris de concevoir de nouveaux manuels et mobilisé à cet effet des spécialistes; ceux-ci ont œuvré dans un contexte de compétition commerciale, ce qui a généré une hétérogénéité dans les choix des terminologies ainsi que dans les critères d'identification des catégories, des groupes et de types de phrases. À titre d'exemple, à la fin des années 70 ont été diffusés quatre manuels ou séries de manuels concurrents: la série Bâtir une grammaire, éditée par Delagrave en 1977 et dirigée par B. Combettes, J. Fresson \& R. Tomassone; la série Nouvel Itinéraire grammatical, éditée la même année par Nathan et dirigée par J. Grunenwald et $\mathrm{H}$. Mitterand ; la série de manuels Français dirigée par C. Cherdon, C. Demols et J. Mottoul et publiée en 1979 par Duculot; la Nouvelle grammaire française de M. Grevisse et A. Goose, publiée l'année suivante par le même éditeur. Comme le montrent les deux tableaux ci-dessous, pour un objet aussi peu complexe que le groupe nominal, les divergences de modes d'analyse et de conceptualisation ont d'emblée été importantes.

Tableau 1. Présentation du groupe nominal dans les deux séries de manuels éditées en France

\begin{tabular}{|c|c|}
\hline Bâtir une grammaire (1977) & Itinéraire grammatical (1977) \\
\hline $\begin{array}{l}\text { a) Le déterminant, mot indispensable au } \\
\text { fonctionnement du groupe nominal; il donne } \\
\text { aussi des renseignements sur le genre et sur le } \\
\text { nombre du nom: } \\
\text { - déterminants définis (articles définis, adjectif } \\
\text { démonstratif, déterminant possessif) } \\
\text { - déterminants indéfinis (articles indéfinis, } \\
\text { autres déterminants indéfinis, et numéraux) } \\
\text { - déterminants partitifs (du, de la, des, etc.) } \\
\text { b) L'expansion du nom } \\
\text { Groupes du GN que l'on peut supprimer: } \\
\text { - un adjectif épithète ou en apposition } \\
\text { - un complément (nom, groupe prép., épithète) } \\
\text { - le nom en apposition } \\
\text { - une proposition relative }\end{array}$ & $\begin{array}{l}\text { a) Les déterminants, petits mots dont la place } \\
\text { obligatoire est en tête du groupe nominal ; deux } \\
\text { déterminants peuvent apparaitre en même } \\
\text { temps devant le nom : } \\
\text { - déterminants définis (article défini, adjectif } \\
\text { démonstratif, adjectif possessif) } \\
\text { - déterminants indéfinis (article indéfini, article } \\
\text { partitif, adjectif indéfini) } \\
\text { b) L'expansion du nom } \\
\text { Le nom peut être accompagné d'un élément qui } \\
\text { est son complétif ou son expansion: } \\
\text { - un adjectif qualificatif } \\
\text { - un nom, complément de nom } \\
\text { - le nom en apposition } \\
\text { - une proposition relative }\end{array}$ \\
\hline
\end{tabular}

Tableau 2. Présentation du groupe nominal dans les manuels édités en Belgique

\begin{tabular}{|l|l|}
\hline Français (1979) & Nouvelle Grammaire française (1980) \\
\hline
\end{tabular}




\begin{tabular}{|c|c|}
\hline $\begin{array}{l}\text { a) Les déterminants, signes simples ou } \\
\text { composés qui, placés devant le nom, } \\
\text { forment avec lui un GN : } \\
\text { - déterminants référents (mon, ce, etc., } \\
\text { renvoient à des éléments du contexte) } \\
\text { - déterminants quantifiants (un, plusieurs, } \\
\text { etc.) } \\
\text { - déterminants qualifiants (certain, tel, } \\
\text { etc.) } \\
\text { b) L'expansion du nom } \\
\text { Un Groupe nominal peut se préciser à l'aide } \\
\text { de trois constituants: } \\
\text { - un adjectif }\end{array}$ & $\begin{array}{l}\text { Éléments subordonnés au nom } \\
\text { a) Les déterminants, ils se joignent à un nom pour le } \\
\text { concrétiser, pour lui permettre de se réaliser dans } \\
\text { une phrase ; espèces : } \\
\text { - articles, définis, indéfinis et partitifs } \\
\text { - numéraux, } \\
\text { - possessifs, } \\
\text { - démonstratifs, } \\
\text { - relatifs, } \\
\text { - interrogatifs et exclamatifs. } \\
\text { b) Épithètes, éventuellement détachées } \\
\text { c) Appositions, éventuellement détachées }\end{array}$ \\
\hline
\end{tabular}
didacticiens ont adressées aux cadres théoriques ayant orienté le mouvement de réforme (en l'occurrence le distributionnalisme bloomfieldien et la grammaire générative). Comme nous l'avons analysé en détail ailleurs (Bronckart, 2008), ces contestations émanaient notamment des travaux de B. Combettes (1983), auteur qui, pour mieux assurer l'articulation des activités grammaticales et des activités d'expression, proposait que les notions issues des analyses syntaxiques soient réexaminées et redécrites dans une perspective fonctionnelle, en termes de thème, rhème, taux d'information, etc. M.-J. Béguelin (2000) a de son côté contesté la pertinence de la notion de phrase et des modalités d'analyse qui en découlent, et proposé un autre corpus notionnel ayant d'évidentes parentés avec celui de B. Combettes. Dans une tout autre perspective enfin, F. Rastier $(2001,2006)$ a soutenu que le paradigme « logicogrammatical» sous-tendant les approches syntaxiques et les grammaires des phrases, était, aux plans épistémologique et méthodologique, radicalement incompatible avec le paradigme « rhétorique/herméneutique » qui serait seul pertinent en matière d'étude du langage. $\mathrm{Si}$, sur le plan de la réflexion théorique, ces contrepropositions étaient légitimes et enrichissantes, projetées comme elles l'ont été sans guère de transposition dans le champ didactique, elles ont de fait participé à une sorte de fuite en avant qui aggrave encore le désordre notionnel.

\section{Du statut de la grammaire}

Dans son acception descriptive qui nous concerne ici, la grammaire se définit comme "l'étude systématique des éléments constitutifs d'une langue", et le statut de la grammaire est dès lors fondamentalement lié à celui que l'on attribue à la langue. LA LANGUE a la réputation de constituer l'objet majeur du structuralisme imputé à $\mathrm{F}$. de Saussure, et depuis les années 50 s'est développé une lecture critique de cette conception présumée qui, en même temps que la langue, discrédite toute approche du système langagier et donc toute grammaire au sens classique de ce terme. En voici deux exemples contemporains particulièrement frappants : 
L'un des objectifs majeurs du structuralisme hérité de Saussure a été de situer la réflexion linguistique dans l'abstraction d'un cadre axiomatiquement fermé, celui de la langue " en soi et pour soi ", en évacuant «l'ensemble hétéroclite des faits de langage ». Et notamment, l'utilisation même du système de la langue, en tant qu'elle implique de prendre en compte des individus parlant, relève des avatars de la parole, et ne constitue pas à ce titre l'objet premier de la linguistique. En d'autres termes, il s'agit d'appréhender la langue en dehors de son usage, ce qui ne peut pas manquer de plonger dans la perplexité quiconque admet que les interactions menées par le biais du langage font cognitivement partie intégrante de ses propriétés, dont elles servent à forger les représentations conceptuelles. (Barbazan, 2008, p. 384)

Le principe du structuralisme en linguistique [hérité du CLG de Saussure] est d'envisager la langue en tant qu'organisation cohérente et autosuffisante - sans rapport donc avec le monde, la pensée, ou une quelconque autre langue - dont les différents éléments (phonologiques, sémantiques, syntaxiques) n'ont de valeur que par les relations strictement internes qu'ils entretiennent les uns avec les autres. Cette approche mécaniste, développée au détriment des facteurs humains et contextuels, considère finalement la langue comme un jeu de construction relativement abstrait dans la mesure où l'on s'intéresse avant tout à son fonctionnement dans l'absolu et guère à son utilisation pratique dans la réalité (la " parole », selon la terminologie saussurienne). (Defays \& Deltour, 2003, p. 36)

21 Les extraits qui précèdent témoignent d'une profonde méconnaissance de l'œuvre réelle de F. de Saussure ${ }^{3}$, dans laquelle on trouve un ensemble de réflexions, argumentées et exemplifiées, donnant un tout autre statut à la langue aussi bien qu'aux pratiques discursives, et débouchant sur une clarification du statut des grammaires et des conditions méthodologiques de leur élaboration.

Comme l'a démontré E. Bulea (2010), la démarche théorique de F. de Saussure se déploie d'abord en une clarification du statut respectif des textes (qualifiés parfois aussi de " parole » ou de « discours ») d'un côté, de la (ou des) langue(s) d'un autre, ainsi que de leurs modes d'interaction. Les notions de textes et de discours sont donc bien présentes dans le corpus saussurien, mais surtout y sont dotées d'une importance capitale, comme le montre l'extrait qui suit :

Toutes les modifications, soit phonétiques, soit grammaticales (analogiques) se font exclusivement dans le discursif. [...] Toute innovation arrive par improvisation, en parlant, et pénètre de là soit dans le trésor intime de l'auditeur ou celui de l'orateur, mais se produit donc à propos du langage discursif. (Saussure, 2002, p. 95)

Ce que souligne par là l'auteur, c'est que les faits langagiers n'existent en tant qu'entités bifaciales que dans l'activité de production ou de réception des textes, et c'est sur la base de cette position qu'il convient d'examiner le statut de la langue, terme qui dans le corpus saussurien a diverses acceptions. Dans les trois que nous retiendrons ${ }^{4}$, ce terme désigne toujours des constructions secondes, élaborées sur base de la pratique et/ou de l'examen des textes.

La première acception, parfois marquée en majuscules (LA LANGUE), parfois qualifiée aussi de "langage ", est d'ordre méthodologique et désigne le produit d'une démarche d'élaboration de concepts et de règles visant à la généralité, sur la base d'un travail d'observation et d'analyse de faits concrets puisés à une diversité d'idiomes :

C'est qu'en effet l'étude du langage comme fait humain est tout entier ou presque tout entier contenu dans l'étude des langues [...]. Langue et langage ne sont qu'une même chose ; l'un est la généralisation de l'autre [...]. Sans cesse par conséquent l'étude générale du langage s'alimente des observations de toutes sortes qui auront été faites dans le champ particulier de telle ou telle langue. (ibid., p. 146) 

p. 65-66)

Les deux autres acceptions portent, non plus sur le processus de construction, mais sur son produit, ou plutôt sur deux types de produits qui se différencient essentiellement par leur lieu d'ancrage, soit dans l'ordre social, soit dans l'ordre individuel, comme il ressort de cette citation contredisant frontalement les affirmations de M. Barbazan et de J.-M. Defays \& S. Deltour :

La conquête de ces dernières années est d'avoir enfin placé [...] tout ce qui est le langage et la langue à son vrai foyer exclusivement dans le sujet parlant soit comme être humain soit comme être social. (op. cit., p. 130)

De manière plus précise, F. de Saussure affirme d'un côté que l'usage des textes/ discours provoque nécessairement en chaque individu une certaine connaissance des entités langagières, de leur statut et de leur organisation :

Tout ce qui est amené sur les lèvres par les besoins du discours, et par une opération particulière, c'est la parole. Tout ce qui est contenu dans le cerveau de l'individu, le dépôt des formes entendues et pratiquées et de leur sens, c'est la langue. [...] La langue est le réservoir individuel; tout ce qui entre dans la langue, c'est-à-dire dans la tête, est individuel. (Saussure, in: Komatsu \& Wolf, 1996,

Il existe donc un état de langue qui est ancré dans le psychisme de chaque personne et qui, en raison de la singularité des parcours d'apprentissage verbal, y est organisé selon des modalités idiosyncrasiques; état que nous avons proposé de qualifier de langue interne (cf. Bronckart, 2010). Mais en d'autres formules, F. de Saussure souligne aussi que « la langue est un ensemble de conventions nécessaires adoptées par le corps social pour permettre l'usage du langage, qui est virtuel chez tout individu» (1968, p. 32). Il signifie de la sorte que des produits de l'usage se déposent aussi dans les représentations collectives et leurs supports (les œuvres et documents) pour y constituer un autre état de langue, d'ordre socioculturel. Ces représentations s'organisent en outre en un savoir à partir duquel s'exerce le contrôle social des productions verbales, sous forme d'évaluation de leur pertinence ou de leur correction eu égard audit savoir, et nous avons proposé en conséquence de qualifier ce second état de langue externe ou normée.

Sur la base de ces apports saussuriens, il est possible tout d'abord de réexaminer le statut des objets de l'enseignement du français, et de considérer que ceux-ci relèvent de trois ordres de phénomènes: les textes/discours, qui exhibent les formes d'organisation de la dimension active, ou praxéologique, du langage ; la langue interne, comme système de représentations des entités langagières ancré dans le psychisme de chaque individu; la langue externe comme ensemble de représentations de ces mêmes entités langagières, telles qu'elles sont véhiculées par les documents du savoir collectif.

Sur cette base encore, il y a lieu d'admettre que les deux niveaux de langue se structurent dans l'ordre gnoséologique, et que leur constitution implique dès lors un processus d'élagage des propriétés spécifiques de l'organisation discursive :

Toute la langue entre d'abord dans notre esprit par le discursif [...]. Mais de même que le son d'un mot, qui est une chose entrée également dans notre for intérieur de cette façon, devient une impression complètement indépendante du discursif, de même notre esprit dégage tout le temps du discursif ce qu'il faut pour ne laisser que le mot. (Saussure, 2002, p. 118)

Sur cette base enfin, il convient de considérer que dans son essence même le langagier est fait d'une circulation permanente entre les trois entités évoquées plus haut: les signes et leurs significations se construisent et se structurent dans l'activité textuelle/ 
discursive ; sous l'effet de l'usage, ils se déposent dans le psychisme individuel, donnant naissance à la langue interne, qui constitue le substrat de l'organisation des représentations individuelles; sous ce même effet, ils se déposent également dans le psychisme collectif, donnant naissance à la langue normée, qui constitue le substrat des représentations collectives.

\section{Que faire en ce domaine aujourd'hui ?} dans le collectif reste dominante la conception des structures linguistiques comme reflets soit de l'organisation des évènements externes, soit de structures cognitives présumées, il s'agirait de faire comprendre enfin qu'une grammaire vise plus modestement à décrire et conceptualiser les règles effectives d'organisation d'une langue naturelle, et non celles de l'intelligence ou des évènements externes. Toujours auprès du public, il s'agirait aussi de faire comprendre que si le travail grammatical implique un effort d'analyse et de conceptualisation, ce travail est néanmoins susceptible de faire apparaitre la relative simplicité et la logique basique des règles du système syntaxique. Auprès des enseignants et de leurs formateurs, il s'agirait de montrer que les grammaires scolaires sont des appareils notionnels constituant une sorte de sas entre la langue interne des apprenants et la langue normée, et que l'apprentissage grammatical vise l'enrichissement et la restructuration positive de cette langue interne. Il s'agirait également de faire admettre que le système grammatical a un mode d'organisation différent de celui des textes/discours, et qu'il y a en conséquence lieu de conserver l'autonomie du travail ayant trait à ce système, dont la conceptualisation doit continuer à relever d'une linguistique des phrases pleinement assumée. tendant à la cohérence. Pour mettre un terme à la cacophonie actuelle, il est impératif de relancer le processus d'harmonisation terminologique qui avait été engagé dans le cadre de l'Association internationale pour la recherche en didactique du français (cf. Chartrand \& De Pietro, 2012). Dans le cadre de ce processus, il y aurait d'abord à élaborer les principes métaméthodologiques sur la base desquels délimiter et définir les diverses rubriques de l'organisation du langagier et expliciter les modalités d'analyse propres à chacune de ces rubriques. Il y aurait aussi à procéder à la stabilisation des notions de grammaire phrastique, en maintenant une ferme distinction entre les plans des classes grammaticales, des syntagmes, des fonctions grammaticales et des valeurs sémantiques. Il y aurait enfin à prendre position sur le statut des formes et mécanismes peu systémiques (modificateurs, apposition, etc.) dont le champ d'application est transphrastique, sinon textuel, et il y aurait ce faisant à expliciter le rapport qu'il convient d'établir entre l'organisation phrastique et les autres modes d'organisation de la langue.

Un troisième chantier a trait aux méthodes d'enseignement de la grammaire et concerne plus spécifiquement les types d'activités qu'il conviendrait de tester et de mettre en œuvre pour développer les capacités d'induction/déduction grammaticale

Pratiques, 169-170 | 2016 
des élèves, et plus largement leurs capacités d'analyse et de raisonnement à propos des faits de langue. Le remarquable numéro 14 de Repères (Vargas \& Grossmann, 1996) comportait un bel ensemble d'articles relatant divers expériences d'innovations de cet ordre, mais dans les ouvrages ou numéros de revue ultérieurs consacrés à la grammaire (cf. notamment Brissaud \& Grossmann, 2009; Nonnon \& Dolz, 2010; Bertrand \& Schaffner, 2013 ; Garcia-Debanc, Paolacci \& Boivin, 2014), si l'on trouve bien quelques articles analysant des expériences d'innovation, la grande majorité des contributions rendent compte de démarches d'analyse des pratiques, qu'il s'agisse de celles des enseignants ou de celles des élèves. Nous aurions tendance à penser que les données issues de ces analyses de pratiques sont aujourd'hui suffisantes, et qu'il conviendrait plutôt de relancer des programmes de recherches visant à concevoir, tester et évaluer une diversité de dispositifs innovants.

Le dernier chantier consisterait en un réexamen des rapports à établir entre l'enseignement grammatical et l'enseignement de l'expression ou des textes. Sur ce plan, nous soutiendrons qu'il convient d'abandonner la hiérarchie qui s'est établie entre finalités de l'enseignement de la langue (selon laquelle la grammaire doit être au service de l'expression) et d'adopter une conception égalitaire, interactionnelle et intégrée de ces finalités. Nous soutiendrons aussi que le travail grammatical devrait conserver un certain taux d'autonomie, se traduisant par l'introduction plus libre d'activités dites "décrochées » et par l'abandon du principe systématique de réexploitation textuelle des acquis grammaticaux. Il s'agirait sur ce plan d'envisager des temps didactiques différents pour le traitement de phénomènes qui pour être interdépendants sont néanmoins de natures différentes. Nous soutiendrons enfin que la formation des enseignants devrait porter sur des dispositifs susceptibles de mettre en œuvre ces démarches de va-et-vient entre travail sur les textes et travail grammatical, dans l'optique d'un réinvestissement réciproque des deux ordres de savoirs construits dans l'une et l'autre démarche.

\section{BIBLIOGRAPHIE}

BARBAZAN, M. (2008). « Principes d'une grammaire prédictive du discours (français langue étrangère et maternelle) ». In : Actes du Congrès mondial de linguistique française. Paris : EDP Sciences, p. 383-396. En ligne : http://dx.doi.org/10.1051/cmlf08115.

BÉGUELIN, M.-J. (2000). De la phrase aux énoncés : grammaire scolaire et descriptions linguistiques. Bruxelles : De Boeck/Duculot.

BERTRAND, O. \& SCHAFFNER, I. (éds) (2013). Enseigner la grammaire. Palaiseau : Éditions de l'École polytechnique.

BESSON, M.-J., BRONCKART, J.-P., CANELAS-TREVISI, S. \& NiCOLAZZI-TURIAN, I. (1990). Français $7^{e}$. Pratique de la langue. Genève : DIP.

BESSON, M.-J., GENOUD, R.-M., LIPP, B. \& NUSSBAUM, R. (1979). Maîtrise du français. Lausanne : Office romand des éditions et du matériel scolaire. 
BRÉAL, M. (1872). Quelques mots sur l'instruction publique en France. Paris : Hachette.

BRISSAUD, C. \& GROSSMANN, F. (éds) (2009). « La construction des savoirs grammaticaux ». Repères, 39. En ligne : https://reperes.revues.org/362.

BRONCKART, J.-P. (2008). « Du texte à la langue, et retour : notes pour une "reconfiguration" de la didactique du français ». Pratiques, 137-138, p. 97-116. En ligne $:$ http://pratiques.revues.org/ 1154.

- (2010). «La vie des signes en questions. Des textes aux langues, et retour ». In : Textos Seleccionados, XXV Encontro Nacional da Associação Portuguesa de Linguística. Porto : APL, p. 11-40. - (2014). « Le rapport du Groupe Bally. Le projet de rénovation de l'enseignement du français en Romandie ». Recherches en didactiques. Les Cahiers Théodile 17, p. 67-91.

BRONCKART, J.-P. \& BESSON, M.-J. (1988). « Et si la grammaire n'était pas inutile ? ». In : Bain, D. et al., La recherche au service de l'enseignement? Genève : CRPP, p. 73-93.

BRONCKART, J.-P., BULEA, E. \& BOTA, C. (éds) (2010). Le projet de Ferdinand de Saussure. Genève : Droz. BRUNOT, F. (1908). L'enseignement de la langue française, ce qu'il est - ce qu'il devrait être dans l'enseignement primaire. Cours de Méthodologie. Paris : Armand Colin.

BULEA, E. (2010). « La langue au service du texte? » Estudos Linguísticos/Linguistics Studies 5, p. 55-76.

BULEA BRONCKART, E. (2014). «Quels repères pour l'enseignement grammatical ? Examen de quelques références actuelles en Suisse romande ». Babylonia 2, p. 36-40.

- (2015). «A grammaire rénovée, exercices... ? ». La Lettre de l'Association internationale pour la recherche en didactique du français (AiRDF) 57, p. 39-45.

CHARTRAND, S. \& DE PIETRO, J.-F. (2012). « Pour une harmonisation des terminologies grammaticales scolaires de la francophonie : quels critères pour quelles finalités ?» Enjeux 84, p. 5-31.

CHERDON, C., DEMOLS, C. \& MOTTOUL, J. (1979). Français 1A. Gembloux : Duculot.

CHISS, J.-L. \& FILLIOLET, J. (1982). « Des changements théoriques dans la linguistique au renouvellement des exercices de grammaire? ». Études de linguistique appliquée 48, p. 46-61.

COMBETtES, B. (1983). Pour une grammaire textuelle. Bruxelles : De Boeck.

COMBETTES, B., FRESSON, J. \& TOMASSONE, R. (1977). Bâtir une grammaire, $6^{e}$. Paris : Delagrave.

DE PIETRO, J.-F. (2010). « L'enseignement grammatical en Suisse francophone : un état des lieux et des questions ». La Lettre de l'AIRDF 45-46, p. 7-17.

DEFAYS, J.-M. \& DELTOUR, S. (2003). Le français langue étrangère et seconde. Enseignement et apprentissage. Liège : Mardaga.

ELALOUF, M.-L. (2014). « La notion de phrase de base dans la pratique des enseignants français : choix terminologiques et enjeux théoriques ». Repères 49, p. 33-55.

FRARY, R. (1884). Manuel du démagogue. Paris : Cerf.

- (1885). La question du latin. Paris : Cerf.

FRANCE, A. (1899). Pierre Nozière. Paris : Calmann-Lévy.

FREINET, C. (1951). Et si la grammaire était inutile. Cannes : Brochures d'éducation nouvelle populaire.

GARCIA-DEBANC, C., PAOLACCI, V. \& Boivin, M.-C. (éds) (2014). « L'étude de la langue : des curricula aux pratiques observées ». Repères 49. En ligne : https://reperes.revues.org/670. 
GENEVAY, E. (1994). Ouvrir la grammaire. Lausanne : LEP.

GREVISSE, M. (1941). Exercices sur la grammaire française. Gembloux : Duculot.

GREVISSE, M. \& GOOSSE, A. (1980). Nouvelle grammaire française. Gembloux : Duculot.

GRUNENWALD, J. \& MITTERAND, H. (1977). Nouvel itinéraire grammatical, $6^{e}$. Paris : Nathan.

HUOT, н. (1981). Enseignement du français et linguistique. Paris : Armand Colin.

KOMATSU, E. \& WOLF, G. (1996). Premier cours de linguistique générale (1907) d'après les cahiers d'Albert

Riedlinger. Oxford : Pergamon.

MASSERON, C. (2001). « Note critique sur les exercices de grammaire ». Pratiques 111-112,

p. 209-236.

Plan de rénovation de l'enseignement du français à l'école élémentaire (1971). Paris : Cahiers de la Fédération de l'Éducation nationale.

RASTIER, F. (2001). Arts et sciences du texte. Paris : Presses universitaires de France.

- (2006). « Formes sémantiques et textualité ». Langages 163, p. 99-114.

ROMIAN, H. (1973). « La recherche-innovation : une problématique complexe ». In : L'enseignement du français à l'école élémentaire. Plan de rénovation. Hypothèses d'action pédagogique. Paris : INRDP, p. 11-28.

ROULET, E. (1972). Théories grammaticales, description et enseignement des langues. Paris : Nathan.

SAUSSURE, F. (de) (1916). Cours de linguistique générale. Paris : Payot.

- (1968). Cours de linguistique générale, t. I. Éd. critique prés. par R. Engler. Wiesbaden :

Harrassowitz.

- (2002). Écrits de linguistique générale. Paris : Gallimard.

VARGAS, C. \& Grossmann, F. (éds) (1996). « La grammaire à l'école. Pourquoi en faire ? Pour quoi faire? ? Repères 14 .

\section{NOTES}

1. Appellation usuelle du Plan de rénovation de l'enseignement du français à l'école élémentaire, adopté en octobre 1970 par une commission présidée par M. Rouchette et publié en 1971.

2. Animé par le signataire, ce Groupe Bally a eu une histoire riche et significative, relatée par J.-P. Bronckart (2014).

3. Nous qualifions d'« œuvre réelle » de F. de Saussure les positions identifiables dans ses notes manuscrites (en partie publiées dans les Écrits de linguistique générale, 2002) et dans les manuscrits des étudiants ayant suivi ses trois cours de linguistique générale ; positions auxquelles le Cours de linguistique générale rédigé par C. Bally \& A. Séchehaye (1916) ne rend que très partiellement justice (cf. Bronckart, Bulea \& Bota, 2010).

4. On trouve en outre, dans le corpus saussurien, une quatrième acception du terme de langue, désignant le «fait de parler » ininterrompu des humains au cours de l'Histoire (cf. Saussure, 2002, p. 156-163). 


\section{RÉSUMÉS}

Ce chapitre propose un bilan de la situation de l'enseignement grammatical dans les pays francophones, et formule un ensemble de propositions, théoriques et didactiques, pour une relance du processus de rénovation en ce domaine. Dans une première partie, nous évoquons d'abord les critiques féroces adressées à l'enseignement grammatical dès l'instauration de l'école publique obligatoire dans le dernier quart $\mathrm{du} \mathrm{XIX}^{\mathrm{e}}$ siècle, puis nous rappelons les principes généraux de la réforme de ce domaine d'enseignement, formulés un siècle plus tard dans le cadre du Plan Rouchette. Nous procédons ensuite à un examen de la situation actuelle de la didactique de la grammaire, qui met en évidence un important désordre terminologique et méthodologique, ainsi qu'une absence de clarté quant à la place et aux objectifs de cet enseignement. En sollicitant les apports de l'œuvre de F. de Saussure, nous procédons alors à un réexamen du statut de la grammaire et de la nature de ses rapports avec le système de la langue d'un côté, avec les textes ou discours d'un autre côté. Et sur la base de ces éléments nous décrivons quatre chantiers qui nous paraissent devoir être mis en œuvre pour une réelle amélioration de la formation grammaticale des élèves et des enseignants.

This chapter provides an overview of the situation of the grammatical teaching in Francophone countries and makes a series of proposals, theoretical and didactic, for a revival of the renovation process in this area. In the first part, we will first evoke fierce criticism of the grammatical teaching since the introduction of public school in the last quarter of the nineteenth, and we recall the general principles of the reform of this area of education, formulated a century later in the Plan Rouchette. We then proceed to an examination of the current situation of the teaching of grammar, which highlights an important terminological and methodological disorder and a lack of clarity about the place and purpose of this teaching. Soliciting inputs from the work of Saussure, we then proceed to a review of the status of grammar and the nature of its relationship with the system of language on one side with texts and discourse on the other hand. And based on these we outline four areas that we think need to be implemented for a real improvement in the grammatical training of students and teachers.

\section{INDEX}

Mots-clés : didactique de la grammaire, didactique de l'expression, manuels, Ferdinand de Saussure, terminologie

Keywords : didactics of grammar, didactics of writing, grammar book, Saussure, terminology

\section{AUTEUR}

JEAN-PAUL BRONCKART

Université de Genève, $\mathrm{CH}-1205$, Suisse 